\title{
Publisher Correction: The discovery of human TSLP as a critical epithelial cytokine in type 2 immunity and allergic disease
}

Vassili Soumelis (iD and Yong-Jun Liu

Correction to: Nature Immunology https://doi.org/10.1038/s41590-020-0720-7, published online 2 July 2020.

In the version of this article initially published, Yong-Jun Liu was not included as a corresponding author. The error has been corrected in the HTML and PDF versions of the article.

Published online: 17 July 2020

https://doi.org/10.1038/s41590-020-0757-7

(c) Springer Nature America, Inc. 2020

\section{Publisher Correction: Tet2 and Tet 3 in B cells are required to repress CD86 and prevent autoimmunity}

Shinya Tanaka, Wataru Ise, Takeshi Inoue, Ayako Ito, Chisato Ono, Yoshihito Shima, Shuhei Sakakibara (D), Manabu Nakayama, Kentaro Fujii, Ikuo Miura, Jafar Sharif, Haruhiko Koseki, Pandelakis A. Koni, Indu Raman, Quan-Zhen Li, Masato Kubo, Katsunori Fujiki, Ryuichiro Nakato (D), Katsuhiko Shirahige, Hiromitsu Araki, Fumihito Miura (D), Takashi Ito DD, Eiryo Kawakami, Yoshihiro Baba (iD) and Tomohiro Kurosaki (iD)

Correction to: Nature Immunology https://doi.org/10.1038/s41590-020-0700-y, published online 22 June 2020.

In the version of this article initially published, in Fig. $1 \mathrm{~d}$, data were omitted from the bDKO CD4 $4^{+}$IFN- $\gamma^{+} \mathrm{T}_{\mathrm{H}} 1$ cell flow cytometry plot (bottom right). The correct Fig. 1d is shown below. In Fig. 8a, the control sample for the H3K9ac and H3K14ac ChIP-seq data (second row from the bottom) was incorrectly labeled "Control 1." The correct label is "Control." The errors have been corrected in the HTML and PDF versions of the article. 